\title{
Laminar Flame Acceleration by Neon-22 Enrichment in White Dwarf Supernovae
}

\author{
David A. Chamulak* ; Edward F. Brown ${ }^{a}$ \\ Michigan State University, Department of Physics and Astronomy and Joint Institute for Nuclear \\ Astrophysics \\ ${ }^{a}$ National Superconducting Cyclotron Laboratory \\ E-mail: chamulak@pa.msu.edu, ebrown@pa.msu.edu
}

\section{Francis X. Timmes}

Thermonuclear Appliations, X-2, Los Alamos National Laboratory

E-mail: timmes@lanl.gov

\begin{abstract}
We explore how the laminar flame speed of degenerate $\mathrm{C} / \mathrm{O}$ thermonuclear burning during a type Ia supernova depends on the composition of the white dwarf. Type Ia supernovae are currently the premier standard candle for measuring distances to redshift $\lesssim 1.6$. The currently favored scenario for this supernovae is the thermonuclear incineration of a $\mathrm{C} / \mathrm{O}$ white dwarf. Recent observations suggest that there may be more than one population of progenitor, and it has been suggested the peak luminosity may depend on the composition of the progenitor white dwarf. Of particular interest is ${ }^{22} \mathrm{Ne}$, which is formed from CNO elements during core He burning of the progenitor star and therefore reflects the metallicity of the progenitor. We find that the laminar flame speed of a C/O mixture increases linearly with the abundance of ${ }^{22} \mathrm{Ne}$ when the abundance of ${ }^{22} \mathrm{Ne}$ is small. The faster and narrower laminar flame enlarges the lengthscale at which turbulent eddies can disrupt the burn. As a result, the addition of ${ }^{22} \mathrm{Ne}$ might lower the density at which a transition to distributed burning occurs.
\end{abstract}

International Symposium on Nuclear Astrophysics - Nuclei in the Cosmos - IX

June 25-30 2006

CERN

\footnotetext{
* Speaker.

$\dagger$ This work was supported by the NSF under grant AST-0507456 and by the Joint Institute for Nuclear Astrophysics at Michigan State University under NSF-PFC grant PHY 02-16783. This work was also funded in part under the auspices of the U.S. Dept. of Energy, and supported by its contract W-7405-ENG-36 to Los Alamos National Laboratory.
} 


\section{Introduction}

In the past decade, type Ia supernovae have become the premier standard candle for measuring the geometry of the universe at redshifts $z \lesssim 1$. Although there is a general belief that the explosion is the thermonuclear incineration of a $\mathrm{C} / \mathrm{O}$ white dwarf that has increased in mass through accretion to just below the Chandrasekhar limit [for a review, see 1], an understanding of the details of the ignition and explosion remain elusive. Of particular importance are whether the explosion is sensitive to properties of the progenitor white dwarf, such as its composition, that depend on the host stellar population. Timmes et al. [2] showed that the mass of ${ }^{56} \mathrm{Ni}$ synthesized in the explosion, which sets the peak brightness, depends linearly on the abundance of ${ }^{22} \mathrm{Ne}$ at densities where electron capture rates are much slower than the explosion timescale, $\sim 1 \mathrm{~s}$. Post-processing of hydrodynamical simulations with embedded tracer particles $[3,4,5]$ have confirmed this dependance. Note that these post-processing calculations used the same (mass fraction $X\left[{ }^{22} \mathrm{Ne}\right]=0$ ) explosion model and only varied the initial composition in the post-processing of the $(\rho, T)$ traces. One dimensional studies that used different progenitor models $[6,7]$ found a much smaller dependence of the ${ }^{56} \mathrm{Ni}$ mass with metallicity, although this may be due to an ambiguity in how the $\mathrm{CNO}$ abundances are scaled relative to $[\mathrm{Fe} / \mathrm{H}]$. It is therefore desirable to explore how variations in the composition of the white dwarf affect the rate of burning during the explosion.

In this contribution, we report on work in progress to calculate how the ${ }^{22} \mathrm{Ne}$ enrichment affects the laminar flame speed, $S_{\text {lam }}$, of a ${ }^{12} \mathrm{C}^{16} \mathrm{O}^{-22} \mathrm{Ne}$ mixture. We compute $S_{\text {lam }}$ as a function of ${ }^{22} \mathrm{Ne}$ abundance for $X\left({ }^{12} \mathrm{C}\right)=0.3$ and 0.7 . Our calculations focus on densities $\rho \geq 10^{8} \mathrm{~g} \mathrm{~cm}^{-3}$. The extension of this work to lower densities, and to other $X\left({ }^{12} \mathrm{C}\right)$ values, will be reported in a forthcoming publication. We begin by briefly describing our how we solve for the flame speed, and then summarize our results. Our central conclusion is that enrichment by a small amount of ${ }^{22} \mathrm{Ne}$ increases $S_{\text {lam }}$ linearly, with a increase in speed of $\approx 30 \%$ for $X\left({ }^{22} \mathrm{Ne}\right)=0.06$. We conclude by briefly describing some implications of our findings.

\section{Model flame calculations}

For our calculations we solved an eigenvalue problem for the conductive flame speed $S_{\text {lam }}$. This method is described in [8], and we shall just give a brief summary here. Under the assumptions of isobaric conditions, the energy equation becomes

$$
S_{\mathrm{lam}}\left[\frac{d E}{d x}+P \frac{d(1 / \rho)}{d x}\right]=\frac{1}{\rho} \frac{d}{d x}\left(K \frac{d T}{d x}\right)+\varepsilon, \quad \varepsilon=N_{\mathrm{A}} \sum_{i} B_{i} \frac{d Y_{i}}{d t}
$$

Here $E$ is the internal energy, $P$ the pressure, $T$ the temperature, $\rho$ the density, $K$ the thermal conductivity; $B_{i}$ and $Y_{i}$ are the binding energy and abundance of species $i$; and $N_{\mathrm{A}}$ is Avogadro's constant. We introduce the heat flux $F=-K d T / d x$ and write the term in square brackets in eq. (2.1) as $-S_{\mathrm{lam}} C_{P} F / K$. This separates the second-order differential equation into two firstorder equations for $T$ and $F$. We relate $d Y_{i} / d t=S_{\text {lam }} d Y_{i} / d x$ and compute $d Y_{i} / d t$ using a reaction network. Our equations neglect mass diffusion, as is appropriate under these conditions.

Equations (2.1), with appropriate boundary conditions, have as an eigenvalue $S_{\text {lam }}$. Ahead of the flame we set the temperature $T_{\text {cold }}=10^{8} \mathrm{~K}$ and $d T / d x$, i.e. the flux $F$, to a small positive value. 
For a trial choice of $S_{\text {lam }}$, the condition to determine the eigenfunction is that $F \rightarrow 0$ asymptotically behind the front and is peaked where $\varepsilon$ is maximum. If a trial $S_{\text {lam }}$ is either too large or too small then the heat flux, F, will diverge from 0 behind the flame front. We solve for $S_{\text {lam }}$ by iterating until $S_{\text {lam }}$ is converged to within $0.01 \%$.

To calculate the heating across the flame front, we use a 430-nuclide network. The reaction rates are taken from the library REACLIB [9]; the light-element rates are mostly experimental and are from the compilation [10]. On the timescale of the flame passage electron captures do not play a role and $Y_{e}$ is essentially fixed. Screening is incorporated using the formalism of Graboske et al. [11]. Use of the 430-nuclide network proved necessary because of the presence of flows to more neutron-rich nuclei when ${ }^{22} \mathrm{Ne}$ is present.

We tested the code by comparing against the results of [8]. We used the same initial composition, 1:1:0 ${ }^{12} \mathrm{C}:{ }^{16} \mathrm{O}:{ }^{22} \mathrm{Ne}$, and the same 130-nuclide set . Overall, our $S_{\text {lam }}$ differ from theirs by no more than $25 \%$ for densities $10^{8}-5 \times 10^{9} \mathrm{~g} \mathrm{~cm}^{-3}$, with the largest discrepancy at $\rho=10^{8} \mathrm{~g} \mathrm{~cm}^{-3}$. We then increased the size of the reaction network to 430 nuclides to assess the effect of network size on flame speed. For this mixture at $\rho=2 \times 10^{9} \mathrm{~g} \mathrm{~cm}^{-3}$, increasing the network from 130 to 430 nuclides increasees $S_{\text {lam }}$ by approximately $25 \%$ even in the absence of ${ }^{22} \mathrm{Ne}$. Further increases in the size of the reaction network did not yield any noticeable change in the flame speed.

\section{Results}

We study how $S_{\text {lam }}$ changed with ${ }^{22} \mathrm{Ne}$ abundance for two different ${ }^{12} \mathrm{C}$ mass fractions: $X\left({ }^{12} \mathrm{C}\right)=$ 0.7 (left panel) and $X\left({ }^{12} \mathrm{C}\right)=0.3$ (right). The remaining composition is composed of ${ }^{16} \mathrm{O}$ and ${ }^{22} \mathrm{Ne}$ with $X\left({ }^{16} \mathrm{O}\right)+X\left({ }^{22} \mathrm{Ne}\right)=1-X\left({ }^{12} \mathrm{C}\right)$. For each fuel mixture, we compute flame speeds for three densities (in the fuel ahead of the flame): $5 \times 10^{8} \mathrm{~g} \mathrm{~cm}^{-3}, 10^{9} \mathrm{~g} \mathrm{~cm}^{-3}$, and $2 \times 10^{9} \mathrm{~g} \mathrm{~cm}^{-3}$. Figure 1 displays the results of our flame calculations with different mixtures of ${ }^{12} \mathrm{C},{ }^{16} \mathrm{O}$, and ${ }^{22} \mathrm{Ne}$. We find that $S_{\text {lam }}$ increases linearly with $X\left({ }^{22} \mathrm{Ne}\right)$ for $0<X\left({ }^{22} \mathrm{Ne}\right)<0.06$ (the total range spanned by these calculation).
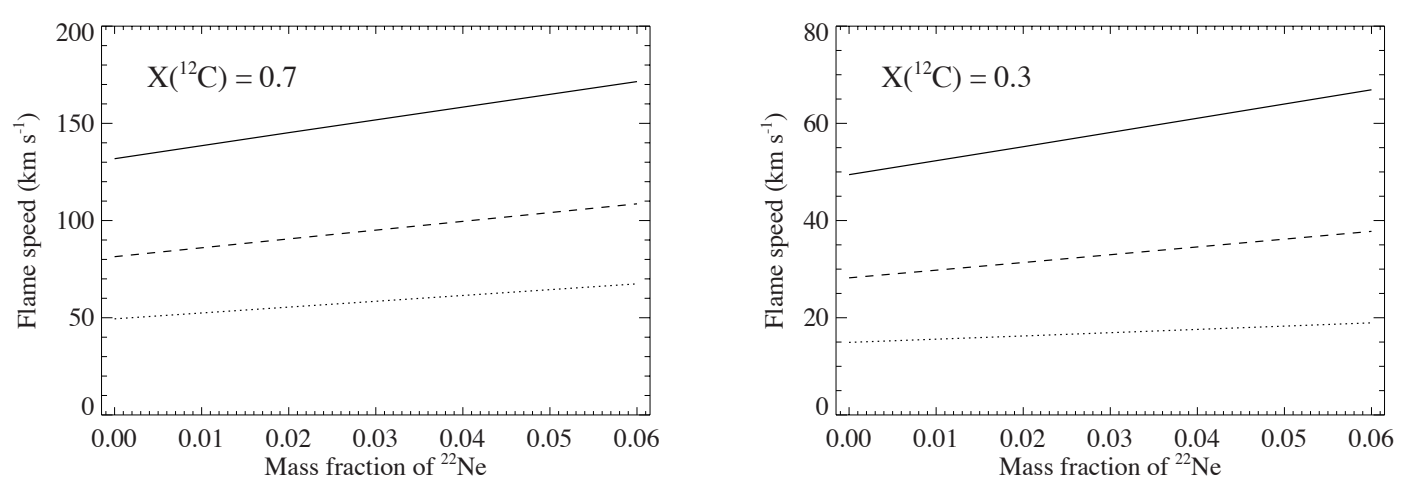

Figure 1: Flame speed as a function of ${ }^{22} \mathrm{Ne}$ for $\mathrm{X}\left({ }^{12} \mathrm{C}\right)=0.7($ left $)$ and $\mathrm{X}\left({ }^{12} \mathrm{C}\right)=0.3($ right $)$. For each family of mixtures, we compute the flame speed for 3 different densities: $2 \times 10^{9} \mathrm{~g} \mathrm{~cm}^{-3}$ (solid line); $10^{9} \mathrm{~g} \mathrm{~cm}^{-3}$ (dashed line); and $5 \times 10^{8} \mathrm{~g} \mathrm{~cm}^{-3}$ (doted line). 
In a $\mathrm{C} / \mathrm{O}$ deflagration, the flame speed and width are set by ${ }^{12} \mathrm{C}$ burning. The buildup of Si-group nuclides and then then establishment of nuclear statistical equilibrium occur on longer timescales, so that the peak of the heat flux is reached where ${ }^{12} \mathrm{C}$ is depleted via the reactions ${ }^{12} \mathrm{C}\left({ }^{12} \mathrm{C}, \alpha\right){ }^{20} \mathrm{Ne}$ and ${ }^{12} \mathrm{C}\left({ }^{12} \mathrm{C}, p\right){ }^{23} \mathrm{Na}(p, \alpha){ }^{20} \mathrm{Ne}$. Once a small abundance of He-nuclei, $X\left({ }^{4} \mathrm{He}\right) \gtrsim$ $10^{-3}$, has accumulated, the lifetime of ${ }^{22} \mathrm{Ne}$ to $(\alpha, n)$ is less than the ${ }^{12} \mathrm{C}$ lifetime. As a result, ${ }^{22} \mathrm{Ne}$ is completely depleted before ${ }^{12} \mathrm{C}$ is, and there is an abundance of free neutrons during ${ }^{12} \mathrm{C}$ burning. This changes the reaction pathways, with the effect that the proton abundance decreases and the ${ }^{4} \mathrm{He}$ abundance increases. We also see a decrease in the abundance of ${ }^{20} \mathrm{Ne}$ in the flame as well. For example, the abundances of ${ }^{17} \mathrm{O}$ and ${ }^{14} \mathrm{C}$ increase via successive $(n, \alpha)$ reactions onto ${ }^{20} \mathrm{Ne}$, until the inverse reactions become significant. Available protons convert ${ }^{14} \mathrm{C}$ to ${ }^{11} \mathrm{~B}$ via ${ }^{14} \mathrm{C}(p, n){ }^{14} \mathrm{~N}(n, \alpha){ }^{11} \mathrm{~B}$. At the temperatures reached in the flame, ${ }^{11} \mathrm{~B}$ is rapidly destroyed via ${ }^{11} \mathrm{~B}(p, 2 \alpha){ }^{4} \mathrm{He}$. Note that the reaction flows just described require two successive neutron captures starting with ${ }^{20} \mathrm{Ne}$. As a result, the rate of additional heating from this pathway is $\propto X\left({ }^{22} \mathrm{Ne}\right)^{2}$ and is also proportional to the rate of ${ }^{12} \mathrm{C}$ burning, which controls the abundance of ${ }^{20} \mathrm{Ne}$. Since $S_{\text {lam }} \sim \varepsilon^{1 / 2}$, this probably explains why the fractional increase in flame speed is linear in $X\left({ }^{22} \mathrm{Ne}\right)$ and does not depend on $X\left({ }^{12} \mathrm{C}\right)$, as shown in Fig. 1. Further work is needed to confirm or refute this conjecture.

\section{Discussion}

Having computed how $S_{\text {lam }}$ of an initially degenerate ${ }^{12} \mathrm{C}-{ }^{16} \mathrm{O}-22 \mathrm{Ne}$ plasma varies with $X\left({ }^{22} \mathrm{Ne}\right)$, we now ask what influence it has on the explosion. There are two places where the laminar flame speed enters. First, during the initial burn near the center of the white dwarf, where the low gravitational acceleration limits the growth of Rayleigh-Taylor instabilities, the flame speed is set by the laminar value. As the front moves outward, the buoyancy of the hot ashes drives RayleighTaylor instabilities that generate turbulence. In this "flamelet" regime, the Gibson length $\ell_{\mathrm{G}}$, defined by $v\left(\ell_{\mathrm{G}}\right)=S_{\text {lam }}$ where $v(\ell)$ is the eddy velocity for a lengthscale $\ell$, is much greater than the laminar flame width $\delta_{\text {lam }}$, and the effective flame propagation speed becomes independent of $S_{\text {lam }}[12,13]$. Here the composition affects the flame speed only through the Atwood number, At $\equiv\left(\rho_{+}-\rho_{-}\right) /\left(\rho_{+}+\rho_{-}\right)$where $\rho_{+(-)}$is the density in the unburnt (burnt) matter. At $\rho \sim 10^{7} \mathrm{~g} \mathrm{~cm}^{-3}$, a transition to distributed burning might occur [14] where $\ell_{\mathrm{G}} \sim \delta_{\text {lam }}$. This is the second place where the increase in $S_{\text {lam }}$ may affect the explosion. For Kolmogorov turbulence, $\ell_{\mathrm{G}} \propto S_{\text {lam }}^{3}$ and hence increasing $S_{\text {lam }}$ by a factor 1.3 (the increase corresponding to changing $X\left({ }^{22} \mathrm{Ne}\right)$ from 0 to 0.06$)$ increases $\ell_{\mathrm{G}}$ by a factor $\approx 2.2$. The enhanced burning rate decreases $\delta_{\text {lam }}$, so that the ratio $\delta_{\mathrm{lam}} / \ell_{\mathrm{G}}$ also decreases with enhanced $X\left({ }^{22} \mathrm{Ne}\right)$; consequently, we expect that the density at which a transition to distributed burning occurs is reduced by the enrichment of ${ }^{22} \mathrm{Ne}$. In a forthcoming publication, we will extend our analysis to lower densities $\rho \sim 10^{7} \mathrm{~g} \mathrm{~cm}^{-3}$ and will study how the change in fuel composition affects At.

\section{References}

[1] W. Hillebrandt and J. C. Niemeyer, Type Ia Supernova Explosion Models, ARA\&A 38 (2000) 191, [astro-ph/0006305]. 
[2] F. X. Timmes, E. F. Brown, and J. W. Truran, On Variations in the Peak Luminosity of Type Ia Supernovae, ApJ 590 (2003) L83, [astro-ph/ 0305114 ].

[3] C. Travaglio, W. Hillebrandt, and M. Reinecke, Metallicity Effect in Multi-Dimensional SNIa Nucleosynthesis, A\&A 443 (2005) 1007, [astro-ph/ 0507510$].$

[4] C. Travaglio, W. Hillebrandt, M. Reinecke, and F.-K. Thielemann, Nucleosynthesis in Multi-Dimensional SN Ia Explosions, A\&A 425 (2004) 1029, [astro-ph/ 0406281 ].

[5] E. F. Brown, A. C. Calder, T. Plewa, P. M. Ricker, K. Robinson, and J. B. Gallagher, Type Ia Supernovae: Simulations and Nucleosynthesis, Nucl. Phys. A 758 (2005) 451, [astro-ph/0505417].

[6] P. Höflich, J. C. Wheeler, and F. K. Thielemann, Type IA Supernovae: Influence of the Initial Composition on the Nucleosynthesis, Light Curves, and Spectra and Consequences for the Determination of Omega M and Lambda, ApJ 495 (1998) 617, [astro-ph/ 9709233 ].

[7] I. Domínguez, P. Höflich, and O. Straniero, Constraints on the Progenitors of Type Ia Supernovae and Implications for the Cosmological Equation of State, ApJ 557 (2001) 279, [astro-ph/0104257].

[8] F. X. Timmes and S. E. Woosley, The Conductive Propagation of Nuclear Flames. I Degenerate $C+O$ and $O+N e+M g$ White Dwarfs, ApJ 396 (1992) 649.

[9] F. Thielemann, M. Arnould, and J. Truran, Thermonuclear Reaction Rates from Statistical Model Calculations, in Advances in Nuclear Astrophysics (E. Vangioni-Flam, J. Audouze, M. Casse, J.-P. Chieze, and J. Tran Thanh Van, eds.), p. 525. Editions Frontières, Gif-sur-Yvette, 1986.

[10] G. R. Caughlan and W. A. Fowler, Thermonuclear Reaction Rates. V., At. Data Nucl. Data Tables 40 (1988), no. 2283.

[11] H. C. Graboske, H. E. Dewitt, A. S. Grossman, and M. S. Cooper, Screening Factors for Nuclear Reactions. II. Intermediate Screening and Astrophysical Applications, ApJ 181 (1973) 457.

[12] A. M. Khokhlov, Propagation of Turbulent Flames in Supernovae, ApJ 449 (1995) 695.

[13] M. Reinecke, W. Hillebrandt, J. C. Niemeyer, R. Klein, and A. Gröbl, A New Model for Deflagration Fronts in Reactive Fluids, A\&A 347 (1999) 724, [astro-ph/9812119].

[14] J. C. Niemeyer and S. E. Woosley, The Thermonuclear Explosion of Chandrasekhar Mass White Dwarfs, ApJ 475 (Feb., 1997) 740, [astro-ph/9607032]. 